\title{
Viabilidade do aproveitamento domiciliar de águas pluviais em municípios de regimes pluviométricos distintos
}

\section{Lívia Veloso Rodrigues, Marcella Piceli Leite, Michael Silveira Thebaldi*, Karina Vilela Rodrigues, Camila Silva Franco e Paula Peixoto Assemany}

Universidade Federal de Lavras. Departamento de Recursos Hídricos e Saneamento. Campus Universitário. Caixa Postal 3037. Lavras-MG, Brasil (CEP 37200-900).*E-mail: michael.thebaldi@ufla.br.

Resumo. Objetivou-se com este estudo analisar a viabilidade técnica e econômica de reservatórios de aproveitamento de água de chuva, dimensionados para três municípios com climas distintos do Estado de Minas Gerais, sendo eles Lavras, Montes Claros e Uberlândia. Para análise da viabilidade técnica, foram usados os métodos de dimensionamento de reservatório apresentados pela NBR 15527: Rippl, Simulação, Azevedo Netto, Alemão, Inglês e Australiano. Para tal, foram utilizadas as séries históricas pluviométricas de cada município para o período de 01/01/1988 a 31/12/2018. A área de captação adotada foi de $100 \mathrm{~m}^{2}$, e foram consideradas demandas de água potável de 100 , 150 e 300 L per capita, em residências ocupadas por três habitantes, sendo o percentual de substituição de água potável por pluvial equivalente a $30 \%$. Para análise da viabilidade econômica, foram utilizadas as metodologias do Payback Descontado e Índice de Lucratividade. Estas foram embasadas nos valores das tarifas praticadas pelas companhias de abastecimento de água e nos custos de aquisição dos reservatórios dimensionados. Os resultados obtidos mostraram que os reservatórios dimensionados por meio do Método da Simulação apresentaram maiores viabilidades técnica em relação aos demais. Com este, a simulação do comportamento dos reservatórios em todos os meses do ano se mostrou eficaz inclusive nos meses de pouca chuva. Ademais, o tempo necessário para retorno do investimento realizado esteve dentro do horizonte de 30 anos adotado, variando de 4,31 a 25,98 anos. 0 investimento também foi considerado vantajoso quando analisados os Índices de Lucratividade, os quais foram todos superiores a um, com valores oscilando de 1,16 a 15,79. Lavras, segunda cidade com maior valor de precipitação média anual dentre as avaliadas, foi a mais viável economicamente para implantação de reservatórios, destacando a tarifa de água e esgoto como um fator de grande relevância na análise.

Recebido

$22 / 04 / 2020$

Aceito

08/12/2020

Disponível on line

$13 / 12 / 2020$

Publicado

31/12/2020

Acesso aberto

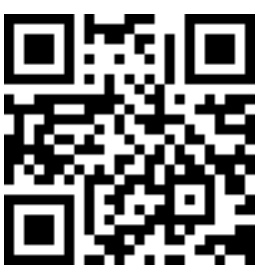

ORCID

(D) 0000-0002-4088-9268 Lívia Veloso Rodrigues

D 0000-0001-7318-4638 Marcella Piceli Leite

(D) 0000-0002-4579-6714 Michael Silveira

Thebaldi

0000-0002-9361-5949

Karina Vilela

Rodrigues

ISSN 2359-1412/RBGAS-2020-0066/2020/7/17/34/1575

Rev. Bras. Gest. Amb. Sustent.

http://revista.ecogestaobrasil.net 
Palavras-chave: Hidrologia urbana; Desenvolvimento sustentável; Reservatório predial de águas pluviais; Viabilidade técnica e econômica.

\section{Abstract. Rainwater harvesting viability at housing buildings} in cities with different rainfall regimes. The objective with this study was to analyze the technical and economic viability of rainwater harvesting reservoirs, designed for three cities of Minas Gerais State (Lavras, Montes Claros and Uberlândia). For technical viability analysis, the methods presented by NBR 15527 were used, being Rippl, Simulation, Azevedo Netto, German, English and Australian. To this end, the historical rainfall series of each city were used for the period from 01/01/1988 to $12 / 31 / 2018$. The adopted harvesting area was $100 \mathrm{~m}^{2}$, and drinking water demands of 100, 150 and $300 \mathrm{~L}$ per capita were considered in houses occupied by three inhabitants, with the percentage of substitution of drinking water for rainwater equivalent to $30 \%$. For economic viability analysis, Discounted Payback and Profitability Index methodologies were used. These were based on the fares charged by the water supply companies and on the acquisition costs of the designed reservoirs. The results showed that the reservoirs dimensioned by the Simulation Method had better technical viability compared to the others. With this one, the simulation of the reservoirs' behavior in every month of the year proved their effectiveness including months of low rainfall. In addition, the time required to return the investment was within the adopted 30 -year horizon, varying from 4.31 to 25.98 years. The investment was also considered profitable when analyzing the Profitability Indexes, which were all higher than one, with values oscillating from 1.16 to 15.79 . Lavras, the second city with the highest average annual precipitation value among the evaluated, was the most economically viable for the implantation of reservoirs, highlighting the water and sewage treatment cost as a great relevance factor in the analysis.

Keywords: Urban hydrology; Rainwater harvesting building reservoir; Sustainability; Technical and economic viability.
D 0000-0002-6756-683X

Camila Silva Franco

(1) 0000-0001-7596-7804

Paula Peixoto

Assemany

\section{Introdução}

As dificuldades de acesso regular, ou mesmo precário, a água ainda é realidade em diversas regiões do mundo, especialmente para famílias e comunidades em situação de extrema pobreza ou localizadas em zonas escassamente povoadas. No entanto, o impacto nos períodos de escassez pode alcançar inclusive grandes centros urbanos, revelando a vulnerabilidade dos sistemas de abastecimento atuais.

A problemática da disponibilidade hídrica em regiões com alto consumo de água, em função das diversas atividades humanas (industriais, agropecuárias e urbanas), sugere a necessidade de potencializar a captação e uso de água. Neste contexto, estudos atuais apresentam novas tecnologias para captação, armazenamento e tratamento de água da 
chuva na escala doméstica (Naddeo et al., 2013; Chao et al., 2015; Liu, 2016; Ghimire et al., 2017; Silva et al., 2019; Wurthmann, 2019).

De acordo com o Diagnóstico dos Serviços de Água e Esgotos (SNIS, 2019), em 2017, o índice médio de perdas na rede de abastecimento e distribuição de água no país foi maior que $35 \%$, sendo que a macrorregião que apresenta melhor resultado é a Centro-Oeste, com $34,1 \%$ de perdas. Essas se dão por meio de vazamentos, erros de medição, fraudes e roubos, além de espelhar os significativos problemas de gestão de recursos hídricos na maior parte das companhias de água e esgoto brasileiras.

Sabe-se que a crise de água não é consequência apenas de fatores ligados a perdas ou empresas de tratamento e distribuição, mas principalmente do uso irracional, pela população. Os dados do último censo (IBGE, 2010) constatam que, cerca de 161 milhões de brasileiros, de uma população perto de 195 milhões, vivem nas cidades. Esse crescimento populacional nos centros urbanos acarreta a exploração dos recursos naturais, particularmente dos recursos hídricos.

Sistemas de captação de água pluvial já são utilizados em maior escala há vários anos em países desenvolvidos, como Alemanha, Austrália, Japão, dentre outros (Dornelles et al., 2012). No Brasil, segundo Teston et al. (2018), regiões de clima semiárido, como alguns estados do Nordeste, por sofrerem com a falta de chuva em algumas épocas do ano, são os que vêm utilizando mais comumente as águas pluviais para fins não potáveis, dos quais se destacam: lavagem de carros, irrigação de jardins, descargas sanitárias e lavagem de áreas impermeabilizadas.

De acordo com Cosentino e Borges (2016) a captação de água pluvial pode ser feita utilizando diferentes meios de captação, como coberturas de telhado, lajes e pisos. No armazenamento podem ser utilizados reservatórios subterrâneos, internos ou externos, e até mesmo a combinação destes.

A implantação de instalações para captação e armazenamento de águas pluviais exige um estudo aprofundado, tendo em vista que o projeto inadequado pode levar ao superdimensionamento dos materiais utilizados. Nesse contexto, destaca-se o reservatório por, na maioria das vezes, ser o item que necessita de um maior dispêndio financeiro e cargas exacerbadas que exigiriam adequações estruturais das edificações ao sistema, podendo tornar a implantação do projeto economicamente inviável, ou até subdimensionados, levando, consequentemente, ao comprometimento do abastecimento (Reis et al., 2019). Assim, é de relevante importância a análise de viabilidade econômica destes investimentos.

A norma NBR 15527:2007 (ABNT, 2007) estabelece metodologia para dimensionamento de reservatórios de águas pluviais para fins não potáveis, utilizando coberturas. No total, esta apresenta seis métodos de cálculo, sendo que dentre estes, três são métodos empíricos e três são baseados em simulações.

Assim, este estudo teve como objetivo analisar a viabilidade técnica e econômica da instalação de reservatório de armazenamento de águas pluviais domiciliar em três municípios do Estado de Minas Gerais de diferentes regimes pluviométricos: Lavras, Montes Claros e Uberlândia, utilizando diferentes métodos de dimensionamento e três demandas por água potável.

\section{Material e métodos}

\section{Áreas estudadas e características pluviométricas}

Os municípios considerados neste estudo estão localizados no estado de Minas Gerais e fazem parte de três diferentes macrorregiões (Sudoeste, Norte e Oeste mineiros). De acordo com dados obtidos por meio do portal do IBGE (2017) as características dos municípios são mostradas na Tabela 1. 
Tabela 1. Caracterização dos Municípios de Lavras, Montes Claros e Uberlândia, MG.

\begin{tabular}{|l|c|c|c|c|c|}
\hline Município & Loc. & Clima & Área $\mathbf{( k m}^{\mathbf{2}}$ ) & Pop. (hab.) & PPC (R\$) \\
\hline Lavras & Sudoeste & $\begin{array}{c}\text { Subtropical } \\
\text { úmido e verão } \\
\text { temperado }\end{array}$ & 564,74 & 103.773 & $22.428,25$ \\
\hline Montes Claros & Norte & $\begin{array}{c}\text { Tropical com } \\
\text { verão seco }\end{array}$ & $3.568,94$ & 409.341 & $21.943,89$ \\
\hline Uberlândia & Oeste & $\begin{array}{c}\text { Tropical com } \\
\text { inverno seco }\end{array}$ & $4.115,21$ & 691.305 & $48.585,36$ \\
\hline
\end{tabular}

Loc. = localização no estado; Pop. = população (habitantes); e PPC = PIB per capita (R\$). Fonte: Adaptado de IBGE (2017) e Alvares et al. (2013).

Os dados pluviométricos mensais e anual de precipitação dos municípios estudados foram obtidos por meio de séries históricas com 30 anos, de dados no período de 01/01/1988 a 31/12/2018. As séries para Lavras, Montes Claros e Uberlândia foram encontradas a partir das estações Usina Couro do Cervo (2145007), São João da Vereda (1644028) e Fazenda Letreiro (1948006), respectivamente. Em seguida, utilizando um software de planilha eletrônica foram obtidas as médias aritméticas dos valores, obtendo a precipitação média mensal de todos os meses do ano, para cada município. Para o cálculo da média anual, utilizou-se a somatória das médias mensais. Esses dados foram coletados por meio do portal HidroWeb, uma ferramenta integrante do Sistema Nacional de Informações sobre Recursos Hídricos (SNIRH, 2018). Por fim, as séries de precipitações médias mensais para cada município podem ser vistos na Tabela 2.

Tabela 2. Precipitações médias mensais e anual dos Municípios de Lavras, Montes Claros e Uberlândia para as séries históricas de 01/01/1988 a 31/12/2018.

\begin{tabular}{|l|c|c|c|}
\hline \multirow{2}{*}{ Mês } & \multicolumn{3}{|c|}{ Precipitação média (mm) } \\
\cline { 2 - 4 } & Lavras & Montes Claros & Uberlândia \\
\hline Janeiro & 290 & 159 & 266 \\
\hline Fevereiro & 170 & 113 & 205 \\
\hline Março & 182 & 129 & 195 \\
\hline Abril & 52 & 43 & 46 \\
\hline Maio & 40 & 9 & 16 \\
\hline Junho & 24 & 3 & 10 \\
\hline Julho & 11 & 3 & 12 \\
\hline Agosto & 13 & 4 & 43 \\
\hline Setembro & 54 & 19 & 107 \\
\hline Outubro & 104 & 64 & 199 \\
\hline Novembro & 172 & 191 & 280 \\
\hline Dezembro & 242 & 219 & 1.466 \\
\hline Média Anual & 1.353 & 955 & \\
\hline
\end{tabular}

\section{Dimensionamento do volume dos reservatórios}

A área de captação considerada foi de $100 \mathrm{~m}^{2}$, valor médio entre os padrões de residências brasileiras. As simulações foram realizadas para as seguintes condições: 
- Demanda diária de água potável de 100, 150 e 300 litros per capita, em residências ocupadas por três moradores;

- Porcentagem de substituição de água potável por pluvial de 30\%; e

- Coeficiente de escoamento superficial igual a 0,80.

Os nove diferentes cenários para o dimensionamento, considerando as condições citadas anteriormente foram definidos: Lavras (1 a 3), Montes Claros (4 a 6) e Uberlândia (7 a 9). Para cada município, os cenários representavam demandas per capita potável de 100,150 e $300 \mathrm{~L}^{-1}$.habitante- ${ }^{1}$. $\mathrm{dia}^{-1}$, em sequência. A demanda mensal potável para cada consumo per capita estipulado foi calculada a partir da Equação 1.

$$
D_{(t)}=\frac{D_{\text {dia }} \cdot N \cdot d}{1000}
$$

em que: $\mathrm{D}_{(\mathrm{t})}=$ demanda mensal potável $\left(\mathrm{m}^{3}\right) ; \mathrm{D}_{\text {dia }}=$ demanda per capita potável ( $\mathrm{L} /$ habitante.dia); $\mathrm{N}=$ quantidade de moradores (habitantes) e $\mathrm{d}=$ número de dias (adotado 30 dias).

Para o cálculo da demanda mensal de água não potável foi considerado um valor de $30 \%$ de $\mathrm{D}_{(\mathrm{t})}$, conforme estabelecido anteriormente.

A demanda anual de água não potável, para cada consumo per capita estipulado, foi calculada a partir da Equação 2, na qual o valor 12 representa o número de meses em um ano. Estes resultados são apresentados na Tabela 3.

$$
\mathrm{D}_{\text {anu al,NP }}=\mathrm{D}_{\text {mensal }, \mathrm{NP}} \cdot 12.1000
$$

em que: $\mathrm{D}_{\text {anual,NP }}=$ demanda anual de água não potável $(\mathrm{L})$ e $\mathrm{D}_{\text {mensal,NP }}=$ demanda mensal de água não potável (L).

Tabela 3. Demandas mensais e anuais de água não potável nos cenários estudados.

\begin{tabular}{|l|c|c|c|}
\hline $\mathbf{D}_{\text {dia }}(\mathbf{L})$ & $\mathbf{D}_{(\mathbf{t})}\left(\mathbf{m}^{\mathbf{3}}\right)$ & $\mathbf{D}_{\text {mensal,NP }}\left(\mathbf{m}^{\mathbf{3}}\right)$ & $\mathbf{D}_{\text {anual,NP }}(\mathbf{L})$ \\
\hline 100 & 9,00 & 2,70 & 32.400 \\
\hline 150 & 13,50 & 4,05 & 48.600 \\
\hline 300 & 27,00 & 8,10 & 97.200 \\
\hline
\end{tabular}

A partir destes dados, foi possível dimensionar o volume dos reservatórios de águas pluviais com base nos métodos apresentados pela NBR 15527 (ABNT, 2007), a saber: Rippl, Simulação e Australiano - baseados em simulações; e Azevedo Netto, Alemão e Inglês - métodos práticos. 0 método de Azevedo Netto requer o número de meses de pouca chuva de cada município, os quais foram definidos a pelos meses que apresentaram precipitação abaixo do valor médio das precipitações mensais. 


\section{Viabilidade econômica}

Para o cálculo do valor a ser pago na fatura de água de cada residência considerada, foram consultadas as tarifas vigentes das companhias de abastecimento de água de cada município. A Companhia de Saneamento de Minas Gerais (COPASA) é a companhia responsável pelo abastecimento de Lavras e Montes Claros e o Departamento Municipal de Água e Esgoto (DMAE) é o órgão encarregado da distribuição de água em Uberlândia. Os valores das tarifas por $\mathrm{m}^{3}$ de água para cada município são mostrados na Tabela 4

Tabela 4. Tarifas de abastecimento de água e esgoto dos Municípios de Lavras, Montes Claros e Uberlândia.

\begin{tabular}{|c|c|c|c|c|}
\hline Município & CIA & $\operatorname{CAT}\left(\mathrm{R} \$ / \mathrm{m}^{3}\right)$ & $\operatorname{Taxa}\left(\mathrm{R} \$ / \mathrm{m}^{3}\right)$ & CT $\left(\mathrm{R} \$ / \mathrm{m}^{3}\right)$ \\
\hline Lavras & COPASA & 3,165 & 3,007 & 6,172 \\
\hline Montes Claros & COPASA & 3,165 & 3,007 & 6,172 \\
\hline Uberlândia & DMAE & 1,65 & 0 & 1,65 \\
\hline
\end{tabular}

CIA = companhia de abastecimento de água; $\mathrm{CAT}=$ custo da água tratada $\left(\mathrm{R} \$ / \mathrm{m}^{3}\right)$; Taxa $=$ taxa de esgoto $\left(\mathrm{R} \$ / \mathrm{m}^{3}\right)$; e $\mathrm{CT}=$ custo total $\left(\mathrm{R} \$ / \mathrm{m}^{3}\right)$. Fonte: adaptado de COPASA (2019) e DMAE Uberlândia (2018).

Em seguida, foram realizadas estimativas de custos de aquisição de reservatórios para armazenamento de água pluvial por meio de pesquisas em oito lojas escolhidas ao acaso no mercado, visando quatro fornecedores distintos e sendo estes fabricados em três materiais: polietileno, filamento contínuo de poliéster e polipropileno. Após a obtenção de preços, foi ajustada a curva de regressão para um modelo matemático potencial de segundo grau que relacionava o custo de aquisição do reservatório em função do volume (Equação 3), em que se obteve um coeficiente de determinação de 0,9467.

$$
\mathrm{C}=10^{-5} \cdot \mathrm{V}^{2}+0,3299 . \mathrm{V}
$$

em que: $\mathrm{C}$ = custo de aquisição do reservatório $(\mathrm{R} \$)$ e $\mathrm{V}$ = volume do reservatório de água pluvial (L).

Na sequência, calculou-se os custos de aquisição dos reservatórios de águas pluviais de acordo com os volumes selecionados. Finalmente, a viabilidade econômica de implantação do sistema foi analisada a partir de dois métodos: Payback Descontado e Índice de Lucratividade, considerando um período de horizonte do investimento de 30 anos, tempo médio de vida útil de tubulações. Para tal, o valor presente líquido foi calculado a partir da Equação 4.

$$
V P L=\frac{F C}{(1+T M A)^{n}}
$$

em que: $\mathrm{VPL}$ = valor presente líquido; $\mathrm{FC}$ = fluxo de caixa; TMA = taxa mínima de atratividade. Foi adotado 6,25\%, correspondente ao valor médio da taxa Selic no ano de 
2019, de acordo com dados disponibilizados pelo Banco Central (BC, 2019) e $n=$ anos analisados.

O fluxo de caixa foi calculado considerando o valor do investimento inicial no ano zero. Nos anos subsequentes, os valores foram relativos à economia na conta de água para cada ano analisado, por exemplo: no ano 1 , o fluxo de caixa era igual a economia na conta referente a um ano de aproveitamento de água pluvial; no ano 2, o valor do fluxo representava a soma do fluxo no ano anterior mais a economia de água referente a um ano de aproveitamento de água pluvial; e assim consecutivamente.

0 retorno de investimento, $\mathrm{R}$, para o ano 1 , foi calculado por meio da soma do investimento inicial e VPL referente a esse ano. Para o ano 2, foi somado o VPL deste ano ao valor de $\mathrm{R}$ referente ao ano anterior, e assim sucessivamente até o ano 30. Posteriormente, o período de retorno do investimento realizado pôde ser calculado a partir da Equação 5.

$$
\mathrm{PB}_{\text {descontado }}=\mathrm{n}_{\text {neg }}-\frac{\mathrm{R}_{\text {neg }}}{\mathrm{VPL}_{\left(\mathrm{n}_{\text {neg }}+1\right)}}
$$

em que: $\mathrm{PB}_{\text {descontado }}=$ tempo para pagar o investimento inicial (anos); $\mathrm{R}_{\text {neg }}=$ último valor de $\mathrm{R}<0$ na série analisada $(\mathrm{R} \$)$; $\mathrm{n}_{\text {neg }}=$ ano referente a $\mathrm{R}_{\text {neg }}$ e $\mathrm{VPL}\left(\mathrm{n}_{\text {neg }}+1\right)=$ valor presente líquido referente ao primeiro valor de $\mathrm{R}>0(\mathrm{R} \$)$.

O segundo método consiste em analisar a relação entre o somatório de VPL do período analisado, e o investimento inicial, e assim mensurar a capacidade do empreendimento de gerar lucro. Se IL > 1, o investimento é considerado vantajoso, sendo que quanto maior, mais rentável este se prova. Por meio da Equação 6 calculou-se o Índice de Lucratividade.

$$
\mathrm{IL}=\frac{\sum \mathrm{VPL}}{\mathrm{I}_{\text {inicial }}}
$$

em que: $\mathrm{IL}=$ índice de lucratividade; $\mathrm{VPL}=$ valor presente líquido $(\mathrm{R} \$)$ e $\mathrm{I}_{\text {inicial }}=$ investimento inicial (R\$).

Neste estudo, para cada cenário, foi feito o somatório do valor presente líquido referente a um período de 30 anos, e o investimento inicial foi o valor equivalente ao custo de aquisição do reservatório. A partir dos resultados, foi possível comparar economicamente e tecnicamente cada uma das situações consideradas e então identificar o método mais eficiente para cada município observado, ou seja, o que obtiver viabilidade assegurada conforme os parâmetros definidos. 


\section{Resultados e discussão}

\section{Análise da viabilidade técnica}

Método de Rippl. Os valores de volumes dos reservatórios de água pluvial obtidos com o uso do método de Rippl, são mostrados na Figura 1, na qual pode-se observar que com o aumento da demanda e consequentemente do balanço hídrico entre $\mathrm{D}_{\text {mensal,NP }}$ e $\mathrm{Q}_{(\mathrm{t})}$, o volume do reservatório também aumentou.

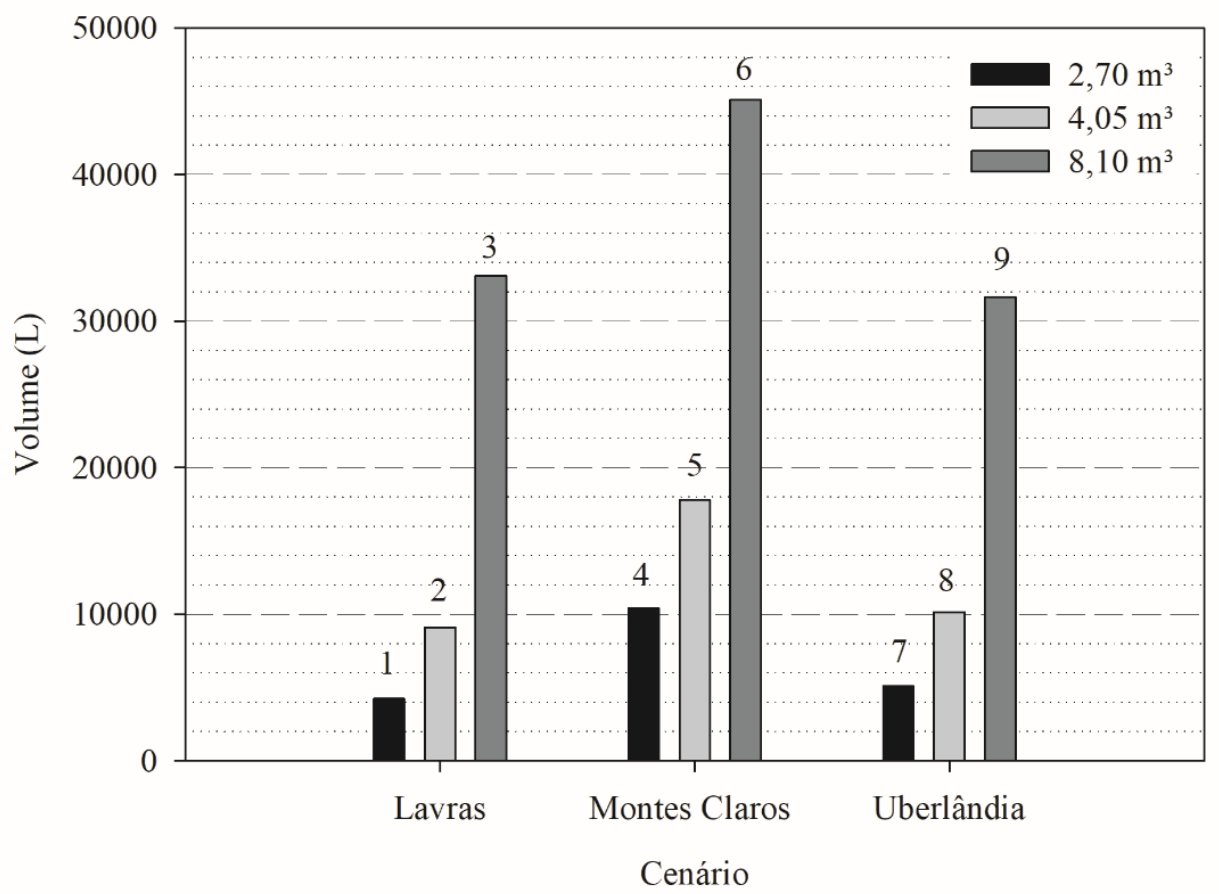

Figura 1. Volumes dos reservatórios para aproveitamento de águas pluviais obtidos por meio do Método de Rippl para os Municípios de Lavras, Montes Claros e Uberlândia, para cada demanda mensal de água não potável.

Para a maioria dos cenários de demandas iguais, o Município de Lavras apresentou os reservatórios com menores volumes, com exceção do cenário 3. Isso se deve ao fato de que o município apresenta menor quantidade de meses em que o volume de chuva não foi capaz de suprir a demanda, ou seja, quando o balanço hídrico assume valor positivo. Além disso, o somatório dos balanços desses meses também foi menor. Esse comportamento não pôde ser observado para o cenário 9, quando o Município de Uberlândia retratou a mesma quantidade de meses com pouca chuva que Lavras, mas menor somatório dos balanços.

Esta situação corrobora com conclusões de Brandão e Marcon (2018), em que a vazão é regularizada através do sistema de acúmulo, no intuito de suprir a demanda de água nos meses com baixo índice de precipitação. 
Método da simulação. As variáveis utilizadas neste método foram as mesmas citadas no método de Rippl, porém, com a adição do volume do reservatório. Assim, os resultados obtidos são mostrados na Figura 2.

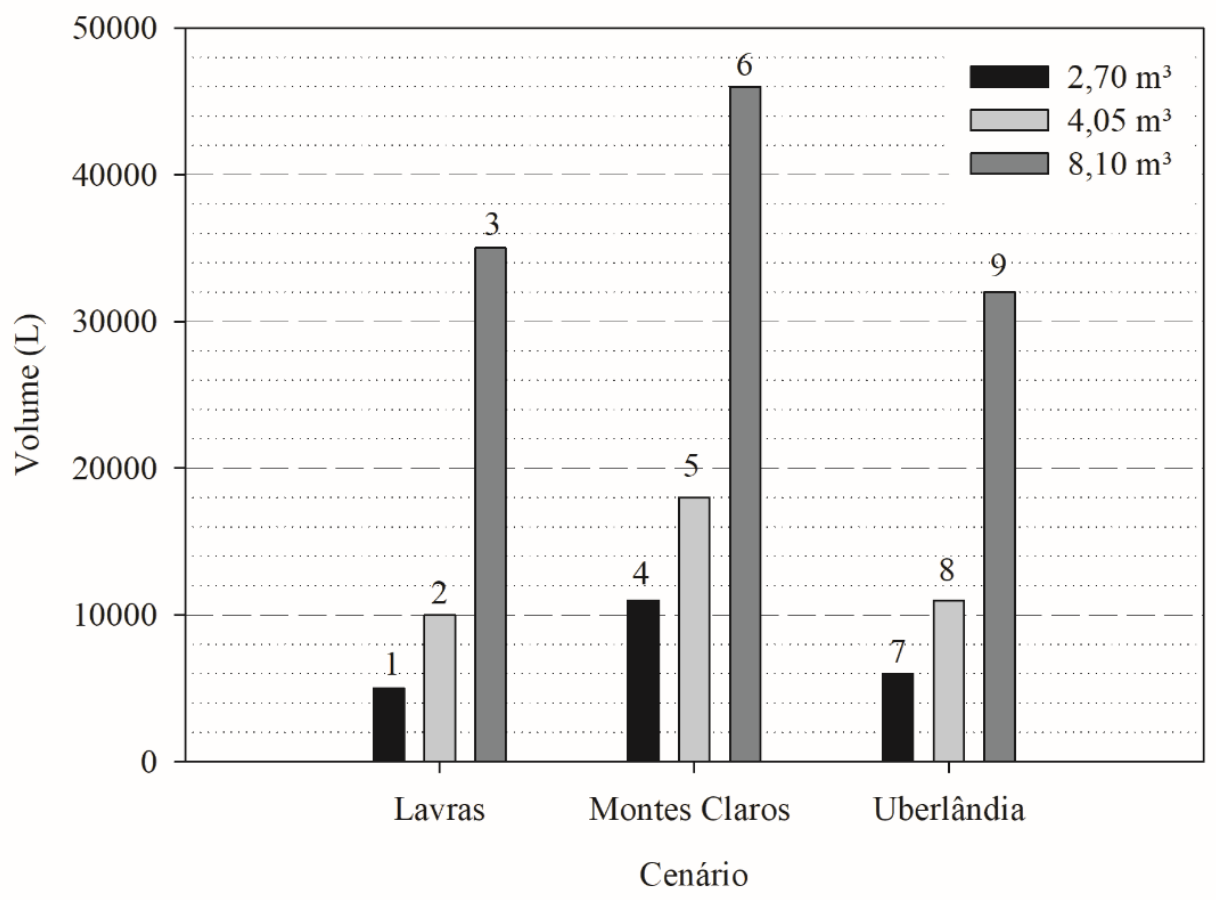

Figura 2. Volumes dos reservatórios para aproveitamento de águas pluviais obtidos por meio do Método da Simulação para os Municípios de Lavras, Montes Claros e Uberlândia, para cada demanda mensal de água não potável.

Por este método foi observado um comportamento semelhante ao Método de Rippl. O Município de Montes Claros demandou reservatórios com volumes superiores, seguido por Uberlândia, cujo volume de reservatório foi inferior apenas para a demanda mensal de $8,1 \mathrm{~m}^{3}$, quando comparado ao Município de Lavras. Neste, o mês de setembro, quando adotado volume de reservatório menor que $35.000 \mathrm{~L}$, necessitou de suprimento de água extra.

Segundo Lopes e Miranda (2016), o Método da Simulação baseia-se em fator pluviométrico, visando um volume ideal de armazenamento que seja capaz de suprir os meses em que há déficit de chuva. Essa é uma das diferenças deste método, quando comparado ao de Rippl.

Método de Azevedo Netto. Os resultados obtidos de volume de reservatório de águas pluviais nos cenários avaliados, com o uso do método de Azevedo Netto, são indicados na Figura 3. 


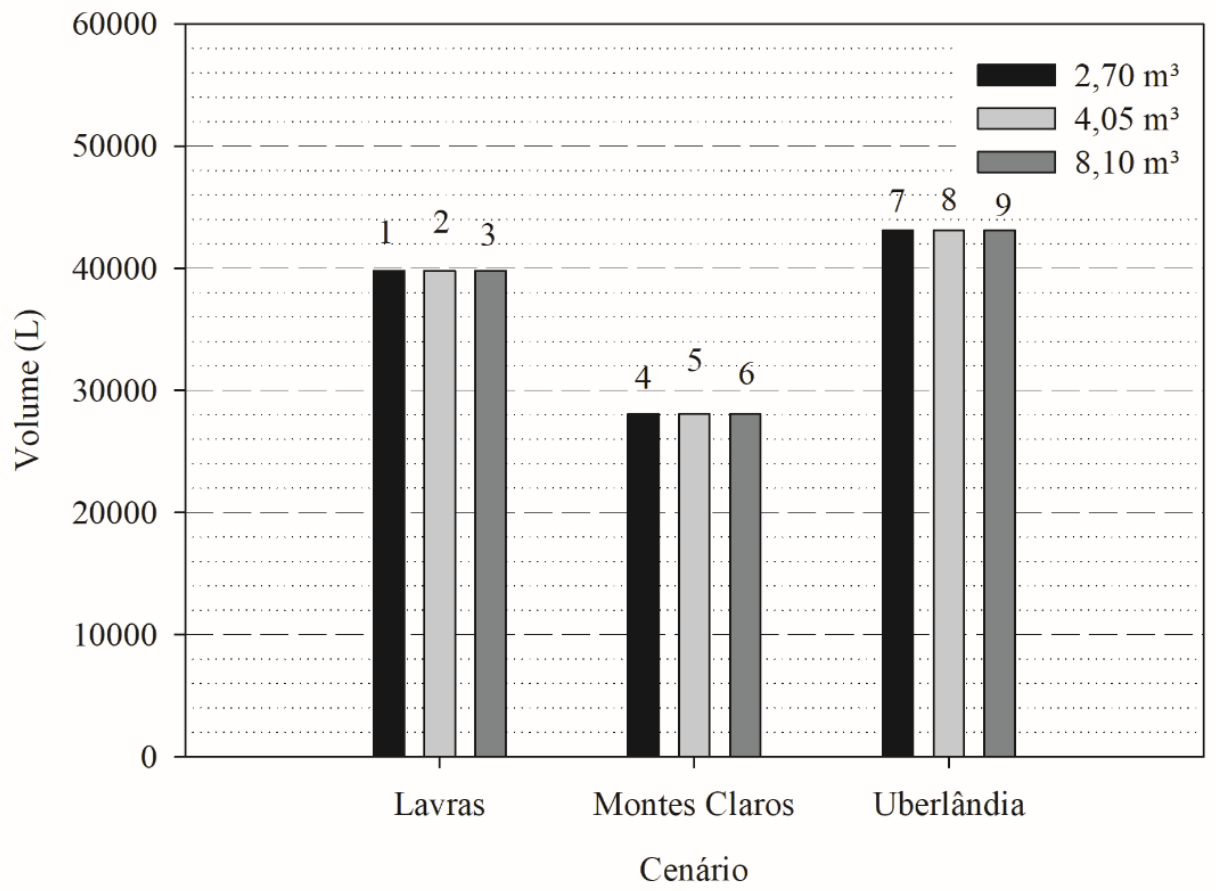

Figura 3. Volumes dos reservatórios para aproveitamento de águas pluviais obtidos por meio do Método de Azevedo Netto para os Municípios de Lavras, Montes Claros e Uberlândia, para cada demanda mensal de água não potável.

Neste método, para todos os valores de $\mathrm{D}_{\text {mensal,NP }}$ foi obtido resultado único para $\mathrm{o}$ volume do reservatório pluvial de cada município, já que se considerou apenas as médias anuais de precipitação e a quantidade de meses com pouca chuva.

Garcia et. al (2018) citam que este método dimensiona o reservatório visando ao armazenamento de água para utilização durante os meses em que o índice de precipitação é menor. Dessa forma, resulta-se em valores elevados de volumes em comparação à outros métodos práticos, devido à quantidade de meses em que a água será armazenada de forma contínua.

O Município de Montes Claros apresentou o menor volume entre os municípios estudados, seguida por Lavras, e posteriormente, Uberlândia, que exigiu o maior reservatório. Este comportamento é proporcional ao volume de chuva anual observado para cada município, ou seja, quanto maior a média de precipitação anual, maior também o volume do reservatório.

Método Prático Alemão. Os resultados obtidos para cada cenário pelo Método Prático Alemão são mostrados na Figura 4. 


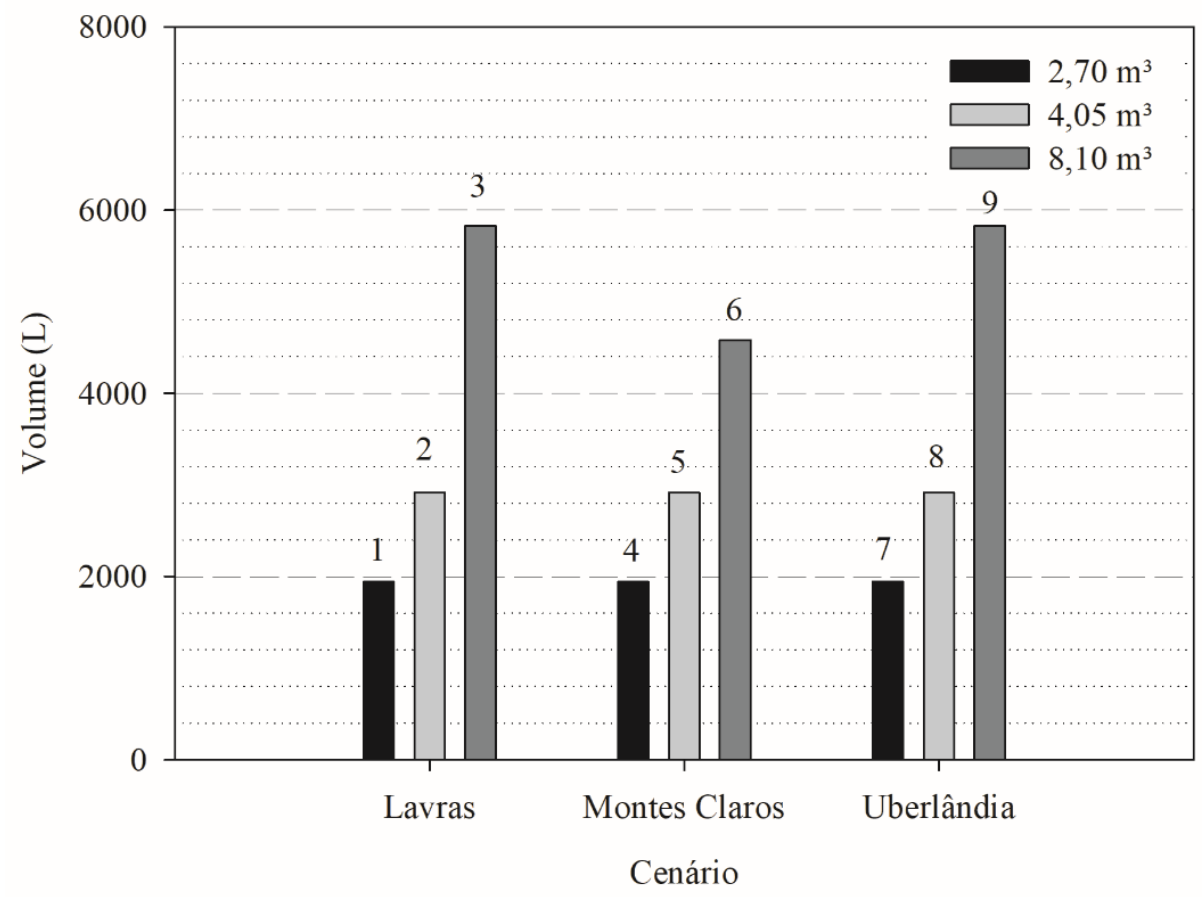

Figura 4. Volumes dos reservatórios para aproveitamento de águas pluviais obtidos por meio do Método Prático Alemão para os Municípios de Lavras, Montes Claros e Uberlândia, para cada demanda mensal de água não potável.

Como este método leva em consideração o menor valor entre $V_{\text {anual }}$ e $D_{\text {mensal, }} \mathrm{NP}$, foram obtidos os mesmos volumes para os cenários que apresentavam as mesmas $\mathrm{D}_{\text {mensal, }}$ NP. Exceção foi encontrada para a demanda de $8,1 \mathrm{~m}^{3}$ do Município de Montes Claros (Cenário 6), único em que o $\mathrm{V}_{\text {anual }}$ foi menor que $\mathrm{D}_{\text {mensal,NP, }}$ devido à baixa precipitação neste município em comparação aos demais. Neste dimensionamento, não se considerou o balanço hídrico entre volume de captação e demanda de água pluvial, o que gerou reservatórios de volumes menores do que as demandas mensais de água não potável. Devido a isto, segundo Souza (2015), reservatórios dimensionados por este método necessitam de fornecimento de água externa, de forma a suprir a demanda.

Método Prático Inglês. Utilizando os valores de precipitação média anual e área de captação foi possível dimensionar os volumes dos reservatórios para cada cenário, sendo os valores apresentados na Figura 5.

Assim como no Método de Azevedo Netto, este também apresentou apenas um valor de volume de reservatório para cada município. Além disso, foi possível observar a proporcionalidade entre a média anual de precipitação e o volume do reservatório, ou seja, quanto maior o valor de precipitação anual para um município, maior será o volume encontrado (Lopes e Miranda, 2016).

O Município de Montes Claros apresentou o menor volume entre os municípios estudados, seguida por Lavras, e depois Uberlândia, que exigiu o maior reservatório. Nesta situação, segundo Brandão e Marcon (2018), o aumento da demanda de água pluvial gera a diminuição da garantia de abastecimento do reservatório. 


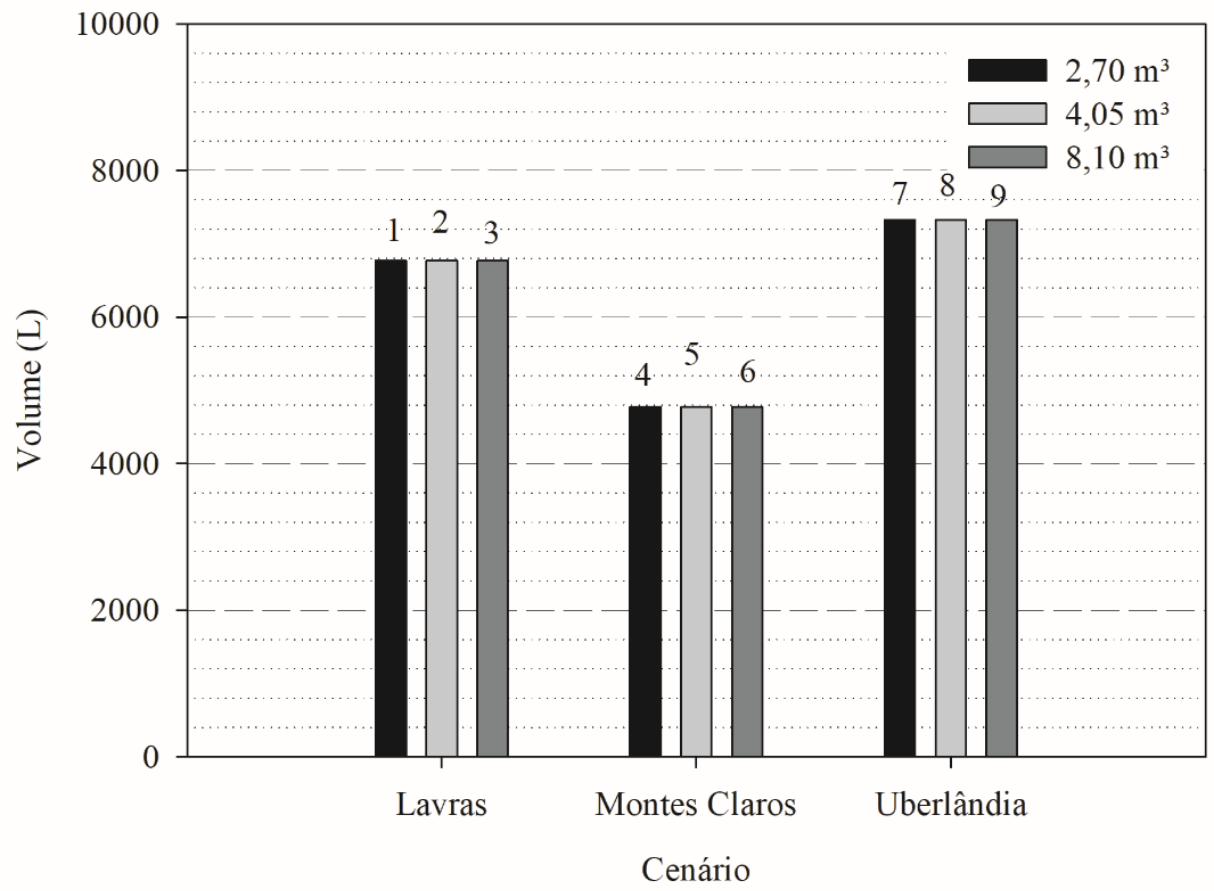

Figura 5. Volumes dos reservatórios para aproveitamento de águas pluviais obtidos por meio do Método Prático Inglês para os Municípios de Lavras, Montes Claros e Uberlândia, para cada demanda mensal de água não potável.

Método Australiano. Os valores para os reservatórios obtidos por meio do Método Australiano para os Municípios de Lavras, Montes Claros e Uberlândia são mostrados na Figura 6.

Para captação a água de chuva no Município de Uberlândia são necessários maiores reservatórios, seguido por Lavras, e posteriormente, Montes Claros. Os reservatórios de maiores volumes foram obtidos nos cenários de menor demanda, devido ao maior valor do balanço hídrico obtido. No cenário 6, não foi possível considerar o reservatório vazio no primeiro mês, já que o nível de confiança prescrito em norma não foi satisfatório, sendo assim necessário o suprimento com água potável, causando aumento no volume do reservatório.

Para este método, verifica-se um armazenamento de toda a água de chuva disponível no mês, e, então, subtrai-se a demanda requerida. Segundo Costa (2016), esse método não garante economia no tamanho do reservatório, uma vez que outros métodos geram como resultado reservatórios de menor volume. De fato, na maioria dos cenários analisados, este foi o método que obteve os maiores volumes.

A partir dos resultados encontrados, foi visualizada grande variação entre os volumes dimensionados por meio de cada método utilizado. Estas também ocorreram em razão dos cenários estudados, os quais tinham como variáveis a demanda de água não potável e o volume de água pluvial, uma vez que foram feitas as análises para três municípios de climas distintos. 


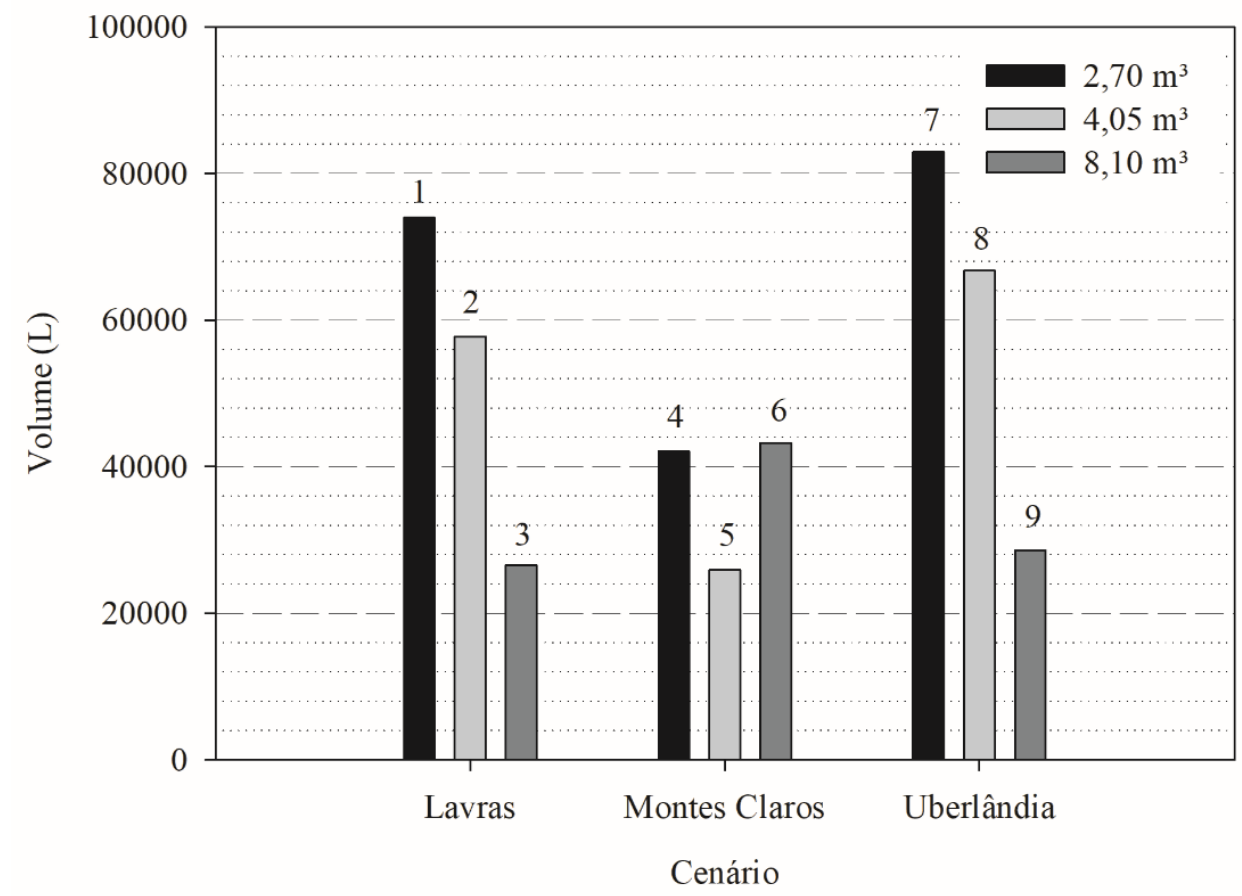

Figura 6. Volumes dos reservatórios para aproveitamento de águas pluviais obtidos por meio do Método Australiano para os Municípios de Lavras, Montes Claros e Uberlândia, para cada demanda mensal de água não potável.

Em aproximadamente 56\% dos cenários, o Método Australiano obteve como resultado os maiores volumes para os reservatórios, e em $100 \%$ dos cenários o Método Alemão trouxe os menores valores. Os métodos de Rippl e Simulação apontaram valores próximos entre si, enquanto os métodos de Azevedo Netto e Inglês, por considerarem nos cálculos apenas as precipitações médias anuais em detrimento às demandas, trouxeram os mesmos volumes para cada município. Assim, na maioria dos cenários, os métodos Australiano e Azevedo Netto superdimensionaram os reservatórios e os métodos Alemão e Inglês subdimensionaram, não atendendo a viabilidade técnica.

Os métodos de Simulação e Rippl trouxeram valores intermediários e razoáveis comparados aos outros métodos. O Método da Simulação simulou o comportamento do volume de água armazenado no reservatório ao longo da série histórica estudada, verificando os meses nos quais o reservatório pré-estabelecido atendeu ou não à demanda de água pluvial. Por este motivo, este foi o método que atendeu o conceito de viabilidade técnica.

\section{Análise da viabilidade econômica}

A viabilidade econômica foi realizada considerando o Método da Simulação, selecionado na análise de viabilidade técnica. 0 valor mensal total a ser pago pelo fornecimento e uso de água correspondente a cada demanda mensal de água potável; a economia mensal que foi obtida na conta, se implantado o aproveitamento da água pluvial; e o valor com reservatório, que representa o valor real a ser pago por meio da utilização do reservatório de águas pluviais, estão expostos na Tabela 5. 
Tabela 5. Valores a serem pagos mensalmente pelo fornecimento e uso de água potável após a instalação do reservatório de águas pluviais nos Municípios de Lavras, Montes Claros e Uberlândia.

\begin{tabular}{|l|c|c|c|c|c|c|}
\hline Município & Cenário & $\mathbf{D}_{(\mathbf{t})} \mathbf{( m}^{\mathbf{3}} \mathbf{)}$ & $\mathbf{D}_{\text {mensal,NP }} \mathbf{( m}^{\mathbf{3}} \mathbf{)}$ & $\mathbf{V M T}(\mathbf{R} \mathbf{)}$ & $\mathbf{E}(\mathbf{R} \mathbf{)}$ & VCR (R\$) \\
\hline \multirow{3}{*}{ Lavras } & 1 & 9,0 & 2,70 & 55,55 & 16,66 & 38,88 \\
\cline { 2 - 7 } & 2 & 13,5 & 4,05 & 83,32 & 25,00 & 58,33 \\
\cline { 2 - 7 } & 3 & 27,0 & 8,10 & 166,64 & 49,99 & 116,65 \\
\hline \multirow{3}{*}{ Montes Claros } & 4 & 9,0 & 2,70 & 55,55 & 16,66 & 38,88 \\
\cline { 2 - 7 } & 5 & 13,5 & 4,05 & 83,32 & 25,00 & 58,33 \\
\cline { 2 - 7 } & 6 & 27,0 & 8,10 & 166,64 & 49,99 & 116,65 \\
\hline \multirow{3}{*}{ Uberlândia } & 7 & 9,0 & 2,70 & 14,85 & 4,46 & 10,40 \\
\cline { 2 - 7 } & 8 & 13,5 & 4,05 & 22,28 & 6,68 & 15,59 \\
\cline { 2 - 7 } & 9 & 27,0 & 8,10 & 44,55 & 13,37 & 31,19 \\
\hline
\end{tabular}

$\mathrm{D}_{(\mathrm{t})}=$ demanda mensal potável; $\mathrm{D}_{\text {mensal,NP }}=$ demanda mensal de água não potável; VMT = valor mensal total; $\mathrm{E}=$ economia mensal na conta; $\mathrm{e} \mathrm{VCR}=$ valor com reservatório.

As tarifas praticadas pela COPASA são superiores às praticadas pelo DMAE. Deste modo, foi observado que a economia no valor da conta de água se fez maior para os Municípios de Lavras e Montes Claros, sendo também proporcional ao valor de $\mathrm{D}_{\text {mensal,NP }}$ em todos os casos analisados. Os custos de aquisição dos reservatórios de águas pluviais, cujos volumes foram definidos pelo Método da Simulação estão apresentados na Tabela 6.

Tabela 6. Custo do reservatório em função do volume dado pelo Método da Simulação para os Municípios de Lavras, Montes Claros e Uberlândia.

\begin{tabular}{|l|c|c|c|}
\hline Município & Cenário & Volume (L) & Custo (R\$) \\
\hline \multirow{3}{*}{ Lavras } & 1 & 5.000 & $1.899,50$ \\
\cline { 2 - 4 } & 2 & 10.000 & $4.299,00$ \\
\cline { 2 - 4 } & 3 & 35.000 & $23.796,50$ \\
\hline \multirow{3}{*}{ Montes Claros } & 4 & 11.000 & $4.838,90$ \\
\cline { 2 - 4 } & 5 & 18.000 & $9.178,20$ \\
\cline { 2 - 4 } & 6 & 46.000 & $36.335,40$ \\
\hline \multirow{3}{*}{ Uberlândia } & 7 & 6.000 & $2.339,40$ \\
\cline { 2 - 4 } & 8 & 11.000 & $2.838,90$ \\
\cline { 2 - 4 } & 9 & 32.000 & $20.796,80$ \\
\hline
\end{tabular}

Foi observado, a partir dos resultados apresentados anteriormente, que os custos dos reservatórios são diretamente proporcionais aos volumes. Porém, esta não é uma relação linear, ou seja, custo e volume não variam a uma taxa constante.

O tempo de retorno do investimento necessário para aquisição dos reservatórios, calculados pelo Método do Payback descontado, de cada cenário, são apresentados na Tabela 7.

De forma geral, os cenários inerentes ao Município de Lavras apresentaram menores tempos de retorno necessários para pagar o investimento, e Uberlândia apresentou os maiores tempos. Isso se deve ao fato de que esse método considera as tarifas aplicadas pelas companhias de abastecimento para calcular o valor presente líquido. As tarifas aplicadas pelo DMAE, órgão responsável pelo abastecimento deste município, são menores do que as tarifas empregadas pela COPASA, companhia de abastecimento dos Municípios de Lavras e Montes Claros. Estas últimas, apesar da 
companhia vigente ser a mesma, apresentaram divergência entre valores. Isso ocorreu devido aos volumes de reservatórios encontrados para cada uma serem diferentes, o que gera variação nos custos de investimento inicial.

Tabela 7. Tempo necessário, em anos, para retorno do investimento inicial realizado nos Municípios de Lavras, Montes Claros e Uberlândia.

\begin{tabular}{|l|c|c|c|c|c|c|}
\hline Município & Cenário & $\mathbf{V ~ ( L ) ~}$ & $\mathbf{n}_{\text {neg }}$ & $\mathbf{R}_{\text {neg }}$ (R\$) & $\mathbf{V P L}_{(\mathbf{n n e g}+\mathbf{1})}$ (R\$) & $\mathbf{P B}_{\text {descontado (anos) }}$ \\
\hline \multirow{4}{*}{ Lavras } & 1 & 5.000 & 4 & $-229,21$ & 738,41 & 4,31 \\
\cline { 2 - 7 } & 2 & 10.000 & 5 & $-685,95$ & $1.250,95$ & 5,55 \\
\cline { 2 - 7 } & 3 & 35.000 & 10 & $-1.965,68$ & $3.387,40$ & 10,58 \\
\hline \multirow{3}{*}{ Montes Claros } & 4 & 11.000 & 7 & $-680,51$ & 984,98 & 7,69 \\
\cline { 2 - 7 } & 5 & 18.000 & 8 & $-1.463,14$ & $1.564,39$ & 8,94 \\
\cline { 2 - 7 } & 6 & 46.000 & 14 & $-498,73$ & $3.624,51$ & 14,14 \\
\cline { 2 - 7 } & 7 & 6.000 & 11 & $-92,15$ & 309,93 & 11,30 \\
\cline { 2 - 7 } & 8 & 11.000 & 14 & $-48,68$ & 484,48 & 14,10 \\
\hline
\end{tabular}

$\mathrm{V}=$ volume do reservatório calculado; $\mathrm{R}_{\text {neg }}=$ último valor de $\mathrm{R}<0$ na série analisada; $\mathrm{n}_{\text {neg }}=$ ano referente a $\mathrm{R}_{\text {neg; }} \mathrm{VPL}\left(\mathrm{n}_{\text {neg }}+{ }_{1)}=\right.$ valor presente líquido referente ao primeiro valor de $\mathrm{R}>0$; $\mathrm{e}$ $\mathrm{PB}_{\text {descontado }}=$ Payback Descontado

Pode-se observar ainda que o sistema se mostrou com uma maior viabilidade econômica quando as tarifas cobradas são mais caras. De acordo com Cruz e Blanco (2017), o preço dos serviços de água e esgoto têm grande influência sobre a viabilidade econômica de implantação de reservatório de aproveitamento de água pluvial, como mostrado neste trabalho. A partir do cálculo do Índice de Lucratividade, para cada cenário, foram obtidos os resultados expostos na Tabela 8.

Tabela 8. Índice de lucratividade do investimento realizado para os Municípios de Lavras, Montes Claros e Uberlândia.

\begin{tabular}{|l|c|c|c|c|c|}
\hline Município & Cenário & $\mathbf{V}(\mathbf{L})$ & $\boldsymbol{\Sigma}$ VPL (R\$) & $\mathbf{I}_{\text {inicial }}$ (R\$) & IL \\
\hline \multirow{3}{*}{ Lavras } & 1 & 5.000 & $29.996,49$ & $1.899,50$ & 15,79 \\
\cline { 2 - 6 } & 2 & 10.000 & $44.994,74$ & $4.299,00$ & 10,47 \\
\cline { 2 - 6 } & 3 & 35.000 & $89.989,47$ & $23.796,50$ & 3,78 \\
\hline \multirow{3}{*}{ Montes Claros } & 4 & 11.000 & $29.996,49$ & $4.838,90$ & 6,20 \\
\cline { 2 - 6 } & 5 & 18.000 & $44.994,74$ & $9.178,20$ & 4,90 \\
\hline \multirow{3}{*}{ Uberlândia } & 6 & 46.000 & $89.989,47$ & $36.335,40$ & 2,48 \\
\cline { 2 - 6 } & 7 & 6.000 & $8.019,15$ & $2.339,40$ & 3,43 \\
\cline { 2 - 6 } & 8 & 11.000 & $12.028,73$ & $4.838,90$ & 2,49 \\
\cline { 2 - 6 } & 9 & 32.000 & $24.057,46$ & $20.796,80$ & 1,16 \\
\hline
\end{tabular}

$\mathrm{V}=$ volume do reservatório calculado; $\sum \mathrm{VPL}=$ somatório do valor presente líquido; $\mathrm{I}_{\text {inicial }}=$ investimento inicial; e IL = índice de lucratividade.

Neste método foi observado um Índice de Lucratividade maior para o Município de Lavras, seguido de Montes Claros e, posteriormente, Uberlândia. Isso ocorreu porque os valores de investimento inicial para o Município de Lavras foram menores, quando 
comparados a Montes Claros. Ao relacionar Uberlândia a Lavras nos cenários de maior demanda, Lavras necessitou de um reservatório de custo maior, porém, mais viável economicamente. Neste caso, o maior Índice de Lucratividade foi devido à maior tarifa de abastecimento em Lavras, gerando maior economia e maior VPL, grandeza diretamente proporcional a esse índice. Consequentemente, este investimento, se realizado em Lavras, se mostra mais vantajoso - um retorno de 15,79 reais para cada real investido no horizonte de análise no cenário de menor demanda por água não-potável.

Neste trabalho, assim como no elaborado por Dias et al. (2007), foi observado que o valor de VPL tende a aumentar com o aumento do volume do reservatório, independentemente do valor da tarifa.

A viabilidade econômica, para o horizonte de projeto adotado de 30 anos, pôde ser verificada para todos os cenários analisados. Contudo, o empreendimento, se implantado no Município de Lavras, apresentará maior viabilidade técnica e econômica quando comparado aos outros dois municípios, mesmo envolvendo custo inicial elevado, no caso de maior demanda.

\section{Conclusão}

O dimensionamento de reservatórios domiciliares de captação de água de chuva foi diretamente influenciado pelo método de dimensionamento e pelos índices pluviométricos apresentados pelos três municípios de climas distintos. O Município de Uberlândia apresenta maior disponibilidade hídrica de captação de água via precipitação. Lavras possui o segundo maior índice pluviométrico, e Montes Claros exibe o pior regime de chuvas em termos de aproveitamento de recursos hídricos, dentre os municípios estudados.

Diante dos métodos de dimensionamento avaliados, o Método da Simulação foi o que melhor atendeu à viabilidade técnica, devido à sua capacidade de simular o comportamento do volume de água reservado ao longo de todos os meses da série histórica adotada, garantindo o suprimento de água não potável até mesmo para os meses de pouca chuva.

Com relação à viabilidade econômica, ambas as metodologias utilizadas, Paypack Descontado e Índice de Lucratividade, indicaram o Município de Lavras, segundo município com maior valor de precipitação média anual, como o município mais viável economicamente para implantação de reservatórios. Por outro lado, o Município de Uberlândia, apesar de apresentar maior volume de precipitação, foi o município com os piores valores de recuperação do investimento e de lucratividade. Destaca-se, portanto, que a tarifa de água e esgoto foi um fator de grande relevância na análise, explicando a maior viabilidade econômica dado o maior preço da tarifa, no caso do Município de Lavras; e a menor viabilidade, devido ao baixo custo tarifa praticada pela companhia de abastecimento de Uberlândia.

Além disso, de acordo com os critérios de avaliação estabelecidos, os reservatórios dimensionados por meio do método de simulação tiveram sua viabilidade técnica e econômica asseguradas, demonstrando a eficácia na economia do consumo de água potável para todos os cenários analisados, e contribuindo para o combate à escassez de recursos hídricos, tanto em município com diferentes regimes pluviométricos e situações de diferentes demandas de água não potável.

Portanto, torna-se válido conferir maior notoriedade a utilização de sistemas de aproveitamento de águas pluviais, uma vez que somente por meio da conscientização e informação este uso será amplamente difundido, o que pode acarretar a economia financeira e redução da vulnerabilidade dos sistemas de abastecimento de água centralizados para atender uma necessidade essencial como o fornecimento de água. 


\section{Conflito de interesses}

Os autores declaram não haver conflito de interesses.

\section{Referências}

ABNT - Associação Brasileira de Normas Técnicas. NBR 15527: Água de chuva Aproveitamento de coberturas em áreas urbanas para fins não potáveis Requisitos. Rio de Janeiro: ABNT, 2007.

Alvares, C. A.; Stape, J. L.; Sentelhas, P. C.; Gonçalves, J. L. M.; Sparovek, G. Köppen's climate classification map for Brazil. Meteorologische Zeitschrift, v. 22, n. 6, p. 711-728, 2013. https://doi.org/10.1127/0941-2948/2013/0507

BC - Banco Central do Brasil. Taxa de juros básica - histórico. Disponível em: <https://www.bcb.gov.br/controleinflacao/historicotaxasjuros>. Acesso em: 12 set. 2019.

Brandão, J. L. B.; Marcon, P. Análise dos métodos de dimensionamento de reservatórios de águas pluviais sugeridos pela NBR 15527/07 com base na simulação diária. Engenharia Sanitária e Ambiental, v. 23, n. 6, p. 1031-1041, 2018. https://doi.org/10.1590/s141341522018129228

Chao, P. R.; Umapathi, S.; Saman, W. Water consumption characteristics at a sustainable residential development with rainwater-sourced hot water supply. Journal of Cleaner Production, v. 109, p. 190-202, 2015. https://doi.org/10.1016/j.jclepro.2015.04.091

COPASA - Companhia de Saneamento de Minas Gerais. Tabela de tarifa. 2019. Disponível em: $\quad<$ http://www.copasa.com.br/wps/wcm/connect/ff53fd67-db13-4d21-992f716d7aa5fecc/Tb_de_tarifa-2018.pdf?MOD=AJPERES\&CACHEID=ff53fd67-db13-4d21992f-716d7aa5fecc>. Acesso em: 26 set. 2019.

Cosentino, L. T.; Borges, M. M. Benefícios da captação de água pluvial para a gestão urbana de recursos hídricos. SBE16 Brazil \& Portugal, Universidade Federal de Juiz de Fora, 2016.

Costa, L. C. Dimensionamento de reservatórios para sistemas de abastecimento de água da chuva para a Região dos Campos Gerais tendo em vista cenários de mudanças climáticas. Ponta Grossa: Universidade Estadual de Ponta Grossa, 2016. (Dissertação de mestrado).

Cruz, W. M.; Blanco, C. J. Aproveitamento de água pluvial para fins não potáveis residenciais em Rio Branco-AC. Perspectivas Online: Exatas \& Engenharias, v. 17, n. 7, p. 12-24, 2017. https://doi.org/10.25242/885X71720171003

Dias, I. C. S.; Athayde Júnior, G. B. A.; Gadelha, C. L. M. Análises da viabilidade econômica de sistemas de aproveitamento de águas pluviais para fins não-potáveis em residências na Cidade de João Pessoa-PB. Revista Econômica do Nordeste, v. 38, n. 4, p. 1-10, 2007.

DMAE - Departamento Municipal de Água e Esgoto. Tarifa de água. 2018. Disponível em: <http://servicos.uberlandia.mg.gov.br/uploads/cms_b_arquivos/19809.pdf>. Acesso em: 26 set. 2019.

Dornelles, F.; Goldenfum, J. A.; Tassi, R. Metodologia para ajuste do fator de esgoto/água para aproveitamento de água de chuva. Revista Brasileira de Recursos Hídricos, v. 17, n. 1, p. 111-121, 2012. https://doi.org/10.21168/rbrh.v17n1.p111-121

Garcia, J. S.; Anjos, N. A. B.; Silva, M. J. M. Determinação do volume de armazenamento de águas pluviais utilizando os Métodos de Azevedo Netto e Prático Inglês. Revista Eletrônica Organizações e Sociedade, v. 7, n. 8, p. 161-171, 2018. 
Ghimire, S. R.; Johnston. J. M.; Ingwersen, W. W.; Sojka, S. Life cycle assessment of a commercial rainwater harvesting system compared with a municipal water supply system. Journal of Cleaner Production, v.151, p. 74-86, 2017. https://doi.org/10.1016/ j.jclepro.2017.02.025

IBGE - Instituto Brasileiro de Geografia e Estatística. Cidades. 2017. Disponível em: <https://cidades.ibge.gov.br/>. Acesso em: 18 nov. 2019.

IBGE - Instituto Brasileiro de Geografia e Estatística. Censo 2010. Disponível em: $<$ https://censo2010.ibge.gov.br/noticias-censo.html?busca=1\&id=3\&idnoticia $=1766 \& \mathrm{t}=$ censo-2010-populacao-brasil-190-732-694-pessoas\&view=noticia>. Acesso em: 26 ago. 2019.

Liu, D. China's sponge cities to soak up rainwater. Nature, v. 537, p. 307, 2016. https://doi.org/10.1038/537307c

Lopes, A. P. G.; Miranda, D. A. Análise crítica de métodos para dimensionamento de reservatórios de água pluvial: estudo comparativo dos Municípios de Belo Horizonte (MG), Recife (PE) e Rio Branco (AC). Scientia cum Industria, v. 4, n. 2, p. 87-91, 2016. https://doi.org/10.18226/23185279.v4iss2p87

Naddeo, V.; Scannapieco, D. Belgiorno, V. Enhanced drinking water supply through harvested rainwater treatment. Journal of Hydrology, v. 498, p. 287-291, 2013. https://doi.org/10.1016/j.jhydrol.2013.06.012

Silva, C. R.; Silva, M. A.; Merlo, M. N.; Thebaldi, M. S. Análise técnica e econômica da captação e uso de águas pluviais em uma edificação com diferentes tipos de coberturas. Revista Brasileira de Gestão Ambiental e Sustentabilidade, v. 6, n. 14, p. 911-930, 2019. https://doi.org/10.21438/rbgas.061420

SNIRH - Sistema Nacional de Informações sobre Recursos Hídricos. 2018. Disponível em: <http://www.snirh.gov.br/hidroweb/publico/medicoes_historicas_abas.jsf>. Acesso em: 10 set. 2019.

SNIS - Sistema Nacional de Informações sobre Saneamento. Diagnóstico dos serviços de água e esgotos. 2017. Disponível em: <http://www.snis.gov.br/diagnostico-agua-eesgotos/diagnostico-ae-2017>. Acesso em: 18 nov. 2019.

Souza, R. L. Análise comparativa dos métodos de dimensionamento de reservatórios para aproveitamento de águas pluviais: estudo de caso Hospital Federal do Andaraí/RJ. Rio de Janeiro: Universidade Federal do Rio de Janeiro, 2015. (Dissertação de mestrado).

Teston, A.; Geraldi, M. S.; Colasio, B. M.; Ghisi, E. Rainwater harvesting in buildings in Brazil: A literature review. Water, v. 10, n. 471, p. 2-25, 2018. https://doi.org/10.3390/ w10040471

Wurthmann, K. A nonparametric bootstrapping method for synthetically generating daily precipitation, water supply, and irrigation demand for rainwater harvesting system storage sizing. MethodsX, v. 6, p. 2669-2676, 2019. https://doi.org/10.1016/ j.mex.2019.10.025

Informação da Licença: Este é um artigo Open Access distribuído sob os termos da Licença Creative Commons Attribution, que permite uso irrestrito, distribuição e reprodução em qualquer meio, desde que a obra original seja devidamente citada. 